Dhaka Univ. J. Biol. Sci. 22(2): 135-144, 2013 (July)

\title{
ROLE OF MATERNAL CORTISOL HORMONE ON GENE REGULATION OF ZEBRAFISH (DANIO RERIO, F. HAMILTON, 1822)
}

\author{
Md. Golam Rabbane*, Mohammad Shamsur Rahman and Anwar Hossain \\ Department of Fisheries, University of Dhaka, Dhaka-1000, Bangladesh
}

Key words: Gene expression, Cortisol hormone, Embryos, Zebrafish

\begin{abstract}
This study was conducted to investigate the role of cortisol, a steroid hormone on the gene expression during embryogenesis of zebrafish (Danio rerio). Gene expression analyses were performed using microarray hybridization. A total 80 genes were found to be up-regulated and 36 genes were down-regulated at $24 \mathrm{hrs}$ post fertilization (hpf) when the fertilized eggs were treated with cortisol hormone. These findings suggest that substantial changes in the responsiveness to maternal cortisol may occur at early developmental phase.
\end{abstract}

\section{Introduction}

The physiological response of a female fish to stress could have considerable consequence in terms of gamete quality and progeny fitness. Egg cortisol contents have been reported in Mozambique tilapia (Oreochromis mossambicus)(1), chum salmon, (Oncorhynchus keta)(2) and other teleosts(3). Specifically, the maternal transfer of cortisol could be modified by physiological processes induced by stress, suggesting that concentrations in eggs could be affected(4). Cortisol concentrations in coho salmon (Oncorhynchus kisutch), eggs were significantly higher when the fish were stressed for 2 weeks prior to spawning(5). McCormick(6) has also published that cortisol administered to ambon damselfish (Pomacentrus ambionensis), resulted in elevated ovarian cortisol concentrations, similar to those of fish experiencing stress in the natural environment. Cortisol implantation into adult tilapia resulted in reduced oocytes size and lower circulating testosterone and $17 \beta$ - estradiol concentrations ${ }^{(7)}$.

On the other hand, no elevation in cortisol concentrations was shown in eggs of rainbow trout that were stressed up to 3 months prior to spawning. In fact, elevated levels of maternal cortisol at ovulation were not reflected in the concentrations in ovarian fluid or eggs (8). Furthermore, embryo of early development has the biochemical machinery to metabolize steroid hormones, including cortisol(9), which suggests that regulation of maternally contributed factors occurs post-fertilization in the embryo. On the basis of these findings, the work was aimed at identifying the effect of cortisol hormone on the gene expression of zebrafish.

*Author for correspondence: <rabbane@univdhaka.edu>. 


\section{Materials and Methods}

Zebrafish (Danio rerio) was used as experimental animal in this study. Immediately after fertilization, fertilized eggs were pooled in cortisol treatment solution and control solution for $2 \mathrm{hrs}$ for development and fixation at $24 \mathrm{hrs}$ post fertilization (hpf). The concentration of cortisol treatment solution was $13 \mu \mathrm{M}$ and the concentration of control solution was $\mathrm{ml}$ abs. ethanol/1 litre fish water. After $2 \mathrm{hrs}$ of treatment, the cortisol treatment and control solution was poured out and eggs were gently washed five-fold in water to eliminate any trace of cortisol and ethanol. Finally the eggs were fixed in liquid nitrogen at $24 \mathrm{hpf}$ to perform RNA extraction analyses.

To evaluate gene expression, total RNA was extracted from cortisol treated and control embryos using Trizol reagent, according to the manufacturer's instructions (Invitrogen, Milan, Italy). At the end of the procedure, the RNA pellet was dried under a chemical hood for 5 - $10 \mathrm{~min}$ and re-dissolved in $12-20 \mu \mathrm{l}$ of RNase-free water. The RNA samples were stored at $-80^{\circ} \mathrm{C}$ until future use.

Highly pure RNA is the key requirement for microarray hybridization, in which $\mathrm{A}_{260} / \mathrm{A}_{230}$ must be higher than 2.2 and $\mathrm{A}_{260} / \mathrm{A}_{280}$ should be higher than 2. Extracted RNA contained different types of contaminants like polysaccharides, carbohydrates, peptides and solvents (phenol, aromatic compounds). These contaminants were removed by $\mathrm{LiCl}$ precipitation.

After $\mathrm{LiCl}$ precipitation, the Agilent 2100 bio-analyzer and RNA LabChip were used to perform RNA quality assessment and quantification. The bio-analyzer software automatically generates the ratio of the $18 \mathrm{~S}$ to $28 \mathrm{~S}$ ribosomal subunits. This ratio plays an important role in determining the level of sample degradation in gel electrophoresis. The Agilent technologies have also introduced a new tool for RNA quality assessment through RNA Integrity Number (RIN).

Two-color Microarray-based gene expression analysis (Agilent Technologies, Santa Clara, CA) was performed to analyze the gene expression of embryos treated with cortisol at $24 \mathrm{hpf}$. The analysis was performed at CRIBI, Italy using Agilent Whole Zebrafish Genome Oligo Microarrays $4 \times 44 \mathrm{~K}$ slide.

The Agilent Feature Extraction Software (FES) was used to read out and process the microarray image files. The software determines feature intensities and ratios (including background subtraction and normalization), rejects outliers and calculates statistical confidences ( $p$ values). Subsequently, the genes were classified into functional groups using gene ontology and analyzed individually at the site "NCBI Gene"(10).

\section{Results and Discussion}

The quality of RNA was controlled by analysing RIN using Agilent 2100 bioanalyzer. RIN was assessed on the base of the presence or absence of degradation 
products in the entire electrophoretic trace of the RNA sample. In this way, interpretation of an electropherogram was facilitated, comparison of samples was enabled and repeatability of experiments was ensured. The results of single analyzed sample were described as an electropherogram and a virtual image of an internal standard agarose gel. Excellent quality of RNA was assessed when the bands of 28S and 18S rRNAs were well separated and their correspondent peaks were in good evidence. RNA samples used in this experiment obtained RIN values 8.6 for control sample (Fig. 1A) and 8.4 for cortisol treated sample (Fig. 1 B). From this result it is clear that RNA quality was good and could be used for further analysis because RIN value greater than 8 is acceptable for microarray hybridization ${ }^{(11)}$.
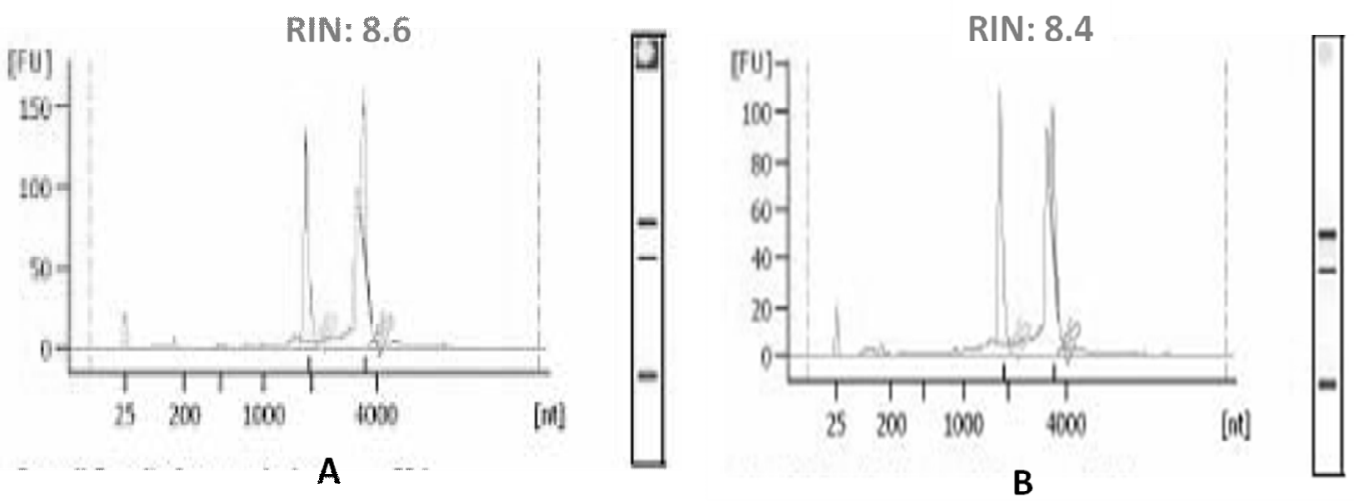

Fig. 1. Electropherogram and virtual gel of quality of RNA samples analyzed by Agilent 2100 Bioanalyzer. The two highest peaks in the electropherogram correspond to $18 \mathrm{~S}$ and $28 \mathrm{~S}$ rRNAs, from left. Axis y describes the fluorescence (FU), while axis $x$ the nucleotide size (nt). 1A: Control and 1B: Cortisol treated samples.

The microarray experiment was carried out according to the experimental design whereas the cRNA quantification and incorporation of Cy3 (green) and Cy5 (red) is shown in Table 1. Treated samples were labeled with Cy5 and the control with Cy3.

Table 1. Quantification of cRNA and incorporation rates of cyanine Cy3 (green) and Cy5 (red) for control and treatment samples, respectively.

\begin{tabular}{lccccc}
\hline Samples & Labeling & $\begin{array}{c}\text { Concentration } \\
\text { cRNA }(\mathrm{ng} / \mu \mathrm{l})\end{array}$ & $\begin{array}{c}\text { Volume } \\
(\mu \mathrm{l})\end{array}$ & $\begin{array}{c}\text { Dye } \\
(\text { picomol} / \mu \mathrm{l})\end{array}$ & $\begin{array}{c}\text { Incorporation } \\
\text { rate }(\mathrm{pmol} / \mu \mathrm{g})\end{array}$ \\
\hline $\begin{array}{l}1.1 \mathrm{Control} \\
24 \mathrm{hpf}\end{array}$ & Cy3 & 296 & 30 & 3.1 & 12.5 \\
\hline $\begin{array}{l}1.1 \mathrm{Cortisol-treated} \\
24 \mathrm{hpf}\end{array}$ & Cy5 & 371 & 30 & 4.5 & 12 \\
\hline
\end{tabular}

High throughput gene expression data from microarray experiments were collected by scanning the signal intensities of the corresponding spots on the array by dedicated fluorescence Agilent's DNA microarray scanner. The spatial distribution of significantly 
up- and down-regulated features is shown in Fig. 2, where red spot indicates that the fluorescence intensity of the Cy5 signal is higher than that of Cy3, which means that the corresponding gene is over-expressed. Green spots indicate that the fluorescence intensity is higher in the control sample than treatment sample, which means that the corresponding gene is down-regulated.

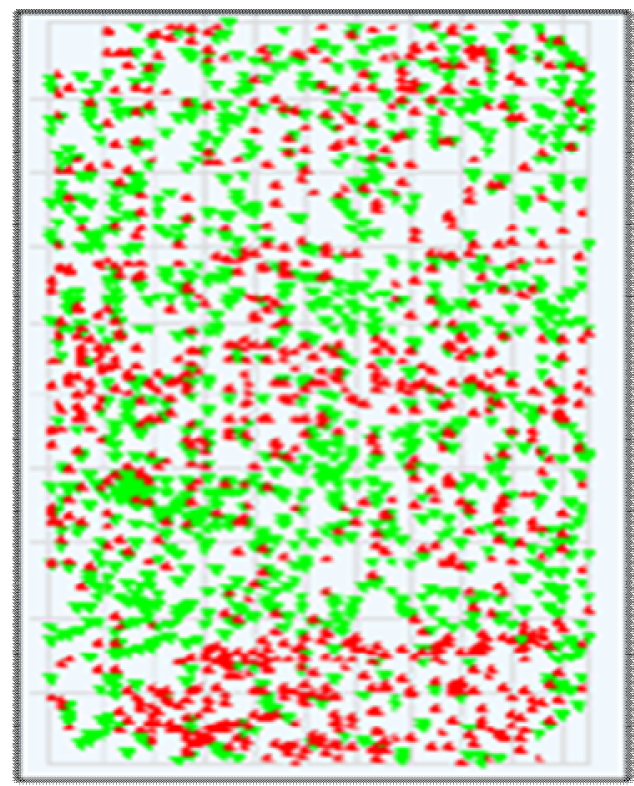

Red: Up-regulated. Green: Down-regulated

Fig. 2. Spatial distribution of significantly up- and down-regulated features.

Image processing was performed using Agilent's Feature Extraction Software (FES) and the normalization of data was performed automatically. This software offers, among other features, the possibility to visualize the results of the data analysis in a log ratio versus log processed signal scatter plot (Fig. 3).

Finally, the up- and down-regulated genes due to cortisol treatment were filtered on the basis of their fold change. Only transcripts that were at least four-fold up- or downregulated were selected. After this selection a total 80 genes were found to be upregulated and only 36 genes were down-regulated. Specific gene description and functions were extracted from public databases "NCBI Gene"(10). The annotations used were derived from Gene Ontology (GO), which provides information on molecular function, as well as from various pathway resources for information on involvement in biological signaling pathways. 


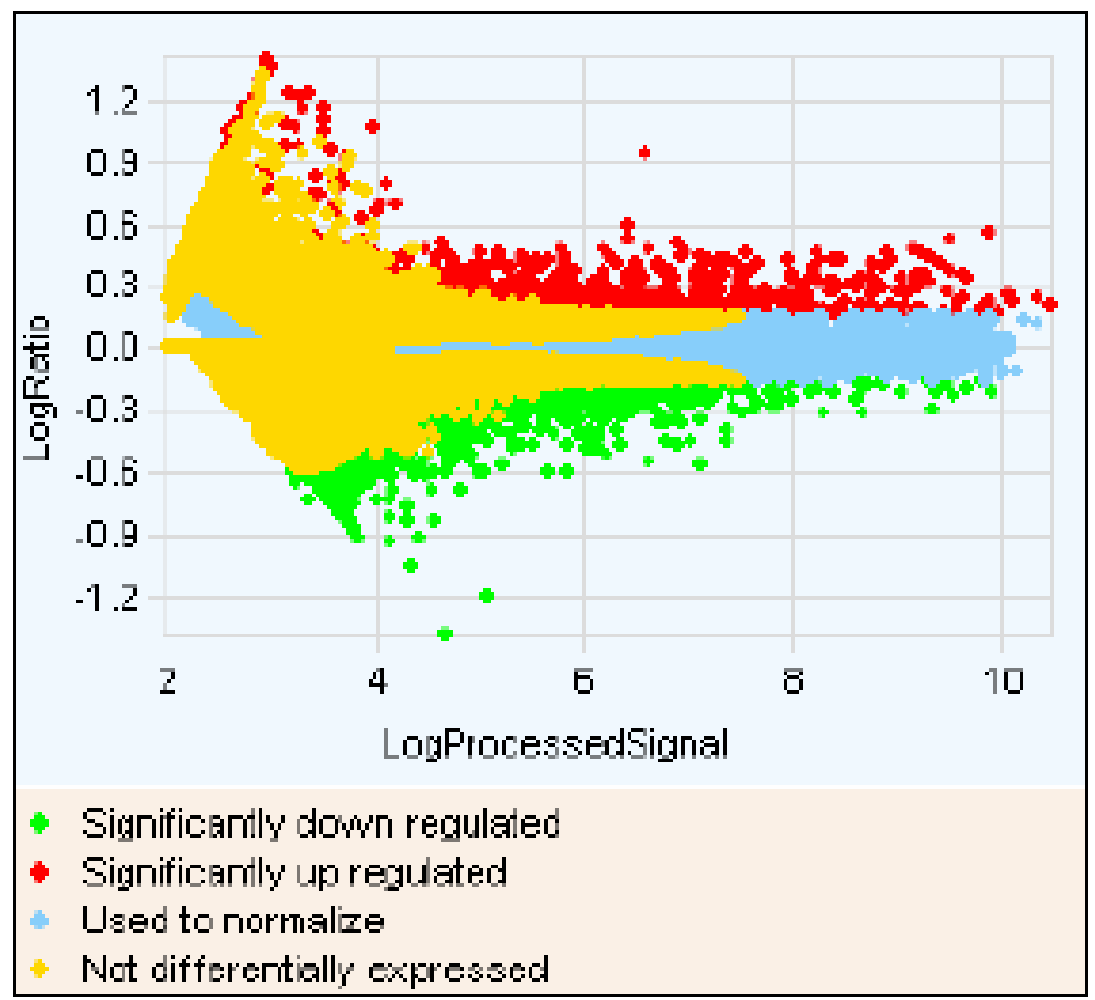

Fig. 3. Scatter plot of log ratio versus log processed signal intensities. Red spot: significantly upregulated genes $(p<0.05)$. Green spot: significantly down-regulated genes $(p<0.05)$. Yellow spot: Not differentially expressed genes. Blue spot: Genes used to normalize.

The up- and down-regulated genes were grouped on the basis of their functions and annotations. The unknown and known function genes that found to be up- and downregulated at $24 \mathrm{hpf}$ are shown in Tables 2 - 4, respectively. The not annotated up- and down- regulated genes are as follows: up- regulated (wu:fe37g12, wu:fc16h11, LOC100002951， zgc:153953， zgc:153629， zgc:153345， zgc:113442， sc:d169， zgc:194392, wu:fd44e01, LOC100149690, zgc:110286, LOC798435, wu:fc45e01 and si:ch211-137c4.4); down- regulated (wu:fc06a09, zgc:66160, zgc:63694, zgc:63694, zgc:171673, si:ch21113c6.2, wu:fc55e03, zgc:171445 and im:7138239). This result is an agreement with the results reported earlier ${ }^{12}$ where 114 and 37 highly expressed transcripts were up- and down-regulated, respectively by knocked down of glucocorticoid receptor mRNA. In addition, intelectin, TLR-5M and TLR-5S mRNA transcripts were poorly and highly expressed at different embryonic stages when oocytes immersed $3 \mathrm{~h}$ prior to fertilization in cortisol-enriched ovarian fluid at either $100 \mathrm{ng} / \mathrm{ml}$ or $1000 \mathrm{ng} / \mathrm{ml}^{(13)}$. 
Table 2. Unknown function up- and down-regulated genes at 24 (hpf) hour post fertilization. Descriptions of genes were extracted from public database "NCBI Gene"(10).

\begin{tabular}{|c|c|c|c|}
\hline $\begin{array}{l}\text { Up-regulated } \\
\text { genes }\end{array}$ & Descriptions & $\begin{array}{l}\text { Down-regulated } \\
\text { genes }\end{array}$ & Descriptions \\
\hline si:ch211-206k20.6 & $\begin{array}{l}\text { BEN domain-containing } \\
\text { protein } 3\end{array}$ & zgc:110152 & chromobox homolog 7a \\
\hline LOC557191 & $\begin{array}{l}\text { guanylate cyclase soluble } \\
\text { subunit beta-2-like }\end{array}$ & LOC568176 & $\begin{array}{l}\text { DCP1 decapping enzyme } \\
\text { homolog B (S. cerevisiae) }\end{array}$ \\
\hline LOC563738 & Fetuin-B-like & ankrd10a & ankyrin repeat domain $10 \mathrm{a}$ \\
\hline $\operatorname{lsm} 8$ & $\begin{array}{l}\text { LSM8 homolog, U6 small } \\
\text { nuclear RNA associated }\end{array}$ & wu:fb11c07 & $\begin{array}{l}\text { Rho GTPase activating } \\
\text { protein } 28\end{array}$ \\
\hline zgc:103632 & thioredoxin-like $4 \mathrm{~A}$ & sumf2 & sulfatase modifying factor 2 \\
\hline LOC557848 & $\begin{array}{l}\text { uncharacterized } \\
\text { LOC557848 }\end{array}$ & zp3 & zona pellucida glycoprotein 3 \\
\hline numb & $\begin{array}{l}\text { numb homolog } \\
\text { (Drosophila) }\end{array}$ & lamb1 & laminin, beta $1 \mathrm{a}$ \\
\hline zgc:174160 & p90 autoantigen & LOC100148642 & $\begin{array}{l}\text { phosphatidylinositol glycan } \\
\text { anchor biosynthesis, class } \mathrm{V}\end{array}$ \\
\hline LOC100002858 & zinc finger protein 850 -like & rpp38 & $\begin{array}{l}\text { ribonuclease PMRP } 38 \\
\text { subunit }\end{array}$ \\
\hline $\operatorname{ttc} 39 c$ & $\begin{array}{l}\text { tetratricopeptide repeat } \\
\text { domain } 39 \mathrm{C}\end{array}$ & wnt8-2 & protein Wnt-8a ORF2 \\
\hline vasp & $\begin{array}{l}\text { vasodilator-stimulated } \\
\text { phosphoprotein }\end{array}$ & & \\
\hline LOC799071 & $\begin{array}{l}\text { aarF domain containing } \\
\text { kinase } 3\end{array}$ & & \\
\hline LOC799923 & FK506-binding protein 15 & & \\
\hline si:ch211-222k6.3 & SLAM family member & & \\
\hline LOC100148935 & $\begin{array}{l}\text { Usher syndrome type-1C } \\
\text { protein-binding protein } 1 \text { - } \\
\text { like }\end{array}$ & & \\
\hline os9 & $\begin{array}{l}\text { amplified in } \\
\text { osteosarcoma }\end{array}$ & & \\
\hline zgc:153456 & NEDD4 binding protein 2 & & \\
\hline jph1a & junctophilin 1a & & \\
\hline $\begin{array}{l}\text { ENSDART000001 } \\
14690\end{array}$ & von Willebrand factor & & \\
\hline and3 & actinodin 3 & & \\
\hline si:dkeyp-84f11.5 & $\begin{array}{l}\text { oxidation resistance } \\
\text { protein } 1\end{array}$ & & \\
\hline
\end{tabular}


Table 3. Up-regulated genes with functions at 24 (hpf) hour post fertilization. Description and functions of genes were extracted from public databases "NCBI Gene"(10)

\begin{tabular}{|c|c|c|}
\hline $\begin{array}{l}\text { Up-regulated } \\
\text { genes }\end{array}$ & Descriptions & Functions \\
\hline zgc:110816 & UPF0364 protein C6orf211 homolog & \\
\hline $\operatorname{cog} 7$ & $\begin{array}{l}\text { component of oligomeric golgi } \\
\text { complex } 7\end{array}$ & \\
\hline sncgb & $\begin{array}{l}\text { synuclein, gamma b (breast cancer- } \\
\text { specific protein } 1 \text { ) }\end{array}$ & \\
\hline zgc:113491 & $\begin{array}{l}\text { PQ-loop repeat-containing protein 2- } \\
\text { like }\end{array}$ & molecular_function \\
\hline fam $46 \mathrm{c}$ & $\begin{array}{l}\text { family with sequence similarity } 46 \text {, } \\
\text { member C }\end{array}$ & \\
\hline sec14l1 & SEC14-like 1 (S. cerevisiae) & \\
\hline ncoa6 & nuclear receptor coactivator 6 & transcription coactivator activity \\
\hline ctsf & cathepsin F & cysteine-type peptidase activity \\
\hline th & tyrosine 3-monooxygenase & $\begin{array}{l}\text { iron ion binding, monooxygenase } \\
\text { activity }\end{array}$ \\
\hline plxnd1 & plexin D1 & Receptor activity \\
\hline bcat1 & $\begin{array}{l}\text { branched chain aminotransferase } 1 \text {, } \\
\text { cytosolic }\end{array}$ & L-isoleucine transaminase activity \\
\hline hsd3b1 & $\begin{array}{l}\text { hydroxy-delta- } 5 \text {-steroid } \\
\text { dehydrogenase, } 3 \text { beta- and steroid } \\
\text { delta-isomerase } 1\end{array}$ & isomerase activity, nucleotide binding \\
\hline ralgapa1 & $\begin{array}{l}\text { ral GTPase-activating protein } \\
\text { subunit alpha-1 }\end{array}$ & GTPase activator activity \\
\hline zgc:153893 & XIAP-associated factor 1 & zinc ion binding \\
\hline hoxa1a & homeo box A1a & $\begin{array}{l}\text { DNA binding, sequence-specific DNA } \\
\text { binding, }\end{array}$ \\
\hline dcc & deleted in colorectal carcinoma & $\begin{array}{l}\text { netrin receptor activity involved in } \\
\text { chemoattraction }\end{array}$ \\
\hline zgc:153452 & $\begin{array}{l}\text { CWC22 spliceosome-associated } \\
\text { protein homolog (S. cerevisiae) }\end{array}$ & DNA , RNA binding \\
\hline msxa & muscle segment homeobox A & $\begin{array}{l}\text { DNA binding, sequence-specific DNA } \\
\text { binding, }\end{array}$ \\
\hline dsel & dermatan sulfate epimerase-like & sulfotransferase activity \\
\hline zgc:154042 & $\begin{array}{l}\text { cytochrome P450, family } 4 \text {, } \\
\text { subfamily V, polypeptide } 7\end{array}$ & iron ion binding, metal ion binding \\
\hline LOC555229 & $\begin{array}{l}\text { sodium-dependent neutral amino } \\
\text { acid transporter B(0)AT3-like }\end{array}$ & \\
\hline
\end{tabular}




\section{(Contd.)}

\begin{tabular}{|c|c|c|}
\hline ehf & ets homologous factor & $\begin{array}{l}\text { DNA binding, sequence-specific DNA } \\
\text { binding, }\end{array}$ \\
\hline ckba & creatine kinase, brain a & $\begin{array}{l}\text { ATP binding, catalytic activity, kinase } \\
\text { activity, }\end{array}$ \\
\hline LOC558601 & pipecolic acid oxidase & oxidoreductase activity \\
\hline $\operatorname{sh} 2 b 1$ & SH2B adaptor protein 1 & $\begin{array}{l}\text { phospholipid binding, signal } \\
\text { transducer activity }\end{array}$ \\
\hline gata2a & GATA-binding protein 2a & sequence-specific DNA binding \\
\hline slc34a2aas & $\begin{array}{l}\text { solute carrier family } 34 \text { (sodium } \\
\text { phosphate), member 2a antisense }\end{array}$ & \\
\hline fynb & $\begin{array}{l}\text { tyrosine-protein kinase fynb, FYN } \\
\text { oncogene related to SRC, FGR, YES b }\end{array}$ & ATP binding, kinase activity \\
\hline zgc:77816 & deoxyribonuclease gamma-like & $\begin{array}{l}\text { deoxyribonuclease activity, } \\
\text { endonuclease activity, }\end{array}$ \\
\hline foxi2 & forkhead box I2 & DNA binding, bending, \\
\hline ctbs & di-N-acetylchitobiase & catalytic activity, chitinase activity, \\
\hline $\operatorname{taf} 1 b$ & $\begin{array}{l}\text { TATA box binding protein (Tbp)- } \\
\text { associated factor, RNA polymerase I, } \\
\text { subunit B }\end{array}$ & DNA binding, \\
\hline ap1s1 & $\begin{array}{l}\text { adaptor-related protein complex } 1 \text {, } \\
\text { sigma } 1 \text { subunit }\end{array}$ & protein transporter activity \\
\hline rsad1 & $\begin{array}{l}\text { radical S-adenosyl methionine } \\
\text { domain containing } 1\end{array}$ & $\begin{array}{l}4 \text { iron, } 4 \text { sulfur cluster binding, } \\
\text { catalytic activity, }\end{array}$ \\
\hline vps8 & $\begin{array}{l}\text { vacuolar protein sorting } 8 \text { homolog } \\
\text { (S. cerevisiae) }\end{array}$ & metal ion binding \\
\hline serac1 & serine active site containing 1 & $\begin{array}{l}\text { hydrolase activity, acting on ester } \\
\text { bonds }\end{array}$ \\
\hline dguok & deoxyguanosine kinase & $\begin{array}{l}\text { ATP binding, phosphotransferase } \\
\text { activity, alcohol group as acceptor }\end{array}$ \\
\hline drp2 & dystrophin related protein 2 & calcium, Zinc ion binding \\
\hline dmrt1 & $\begin{array}{l}\text { doublesex and mab- } 3 \text { related } \\
\text { transcription factor } 1\end{array}$ & $\begin{array}{l}\text { DNA binding, metal ion binding, } \\
\text { sequence-specific DNA binding }\end{array}$ \\
\hline chrna2b & $\begin{array}{l}\text { cholinergic receptor, nicotinic, alpha } \\
\text { polypeptide } 2 \mathrm{~b} \text { (neuronal) }\end{array}$ & $\begin{array}{l}\text { extracellular ligand-gated ion channel } \\
\text { activity, ion channel activity }\end{array}$ \\
\hline pfn2l & profilin 2 like & actin binding \\
\hline stau1 & $\begin{array}{l}\text { staufen, RNA binding protein, } \\
\text { homolog } 1 \text { (Drosophila) }\end{array}$ & $\begin{array}{l}\text { RNA binding, double-stranded RNA } \\
\text { binding }\end{array}$ \\
\hline ube2r2 & ubiquitin-conjugating enzyme E2R 2 & acid-amino acid ligase activity \\
\hline ust & uronyl-2-sulfotransferase & $\begin{array}{l}\text { sulfotransferase activity, transferase } \\
\text { activity }\end{array}$ \\
\hline
\end{tabular}


Table 4. Down-regulated genes with functions at 24 (hpf) hour post fertilization. Description and functions of genes were extracted from public databases "NCBI Gene"(10).

\begin{tabular}{|c|c|c|}
\hline $\begin{array}{l}\text { Down-regulated } \\
\text { genes }\end{array}$ & Descriptions & Functions \\
\hline ube2q2 & $\begin{array}{l}\text { ubiquitin-conjugating enzyme } \\
\text { E2Q family member } 2\end{array}$ & acid-amino acid ligase activity \\
\hline scyl3 & $\begin{array}{l}\text { SCY1-like } 3 \text { (S. cerevisiae), } \\
\text { protein-associating with the } \\
\text { carboxyl-terminal domain of ezrin }\end{array}$ & ATP binding, protein kinase activity, \\
\hline zgc:173443 & fish-egg lectin-like & molecular function \\
\hline tead1a & TEA domain family member $1 \mathrm{a}$ & $\begin{array}{l}\text { sequence-specific DNA binding } \\
\text { transcription factor activity }\end{array}$ \\
\hline wnt8a & $\begin{array}{l}\text { wingless-type MMTV integration } \\
\text { site family, member } 8 \text { a }\end{array}$ & G-protein coupled receptor binding \\
\hline zgc:154116 & beta-1,4-galactosyltransferase 1 & $\begin{array}{l}\text { lactose synthase activity, transferase } \\
\text { activity, }\end{array}$ \\
\hline ppm1g & $\begin{array}{l}\text { protein phosphatase } 1 \mathrm{G} \text { (formerly } \\
2 \mathrm{C} \text { ), magnesium-dependent, } \\
\text { gamma isoform }\end{array}$ & $\begin{array}{l}\text { catalytic activity, hydrolase activity, } \\
\text { metal ion binding, }\end{array}$ \\
\hline zgc:162964 & Golgi resident protein GCP60 & fatty-acyl-CoA binding \\
\hline rbbp61 & retinoblastoma binding protein 6 & $\begin{array}{l}\text { metal ion binding, nucleic acid binding, } \\
\text { zinc ion binding }\end{array}$ \\
\hline $\operatorname{eg} \ln 3$ & egl nine homolog 3 & $\begin{array}{l}\text { L-ascorbic acid binding, iron ion } \\
\text { binding, oxidoreductase activity, }\end{array}$ \\
\hline rem1 & $\begin{array}{l}\text { rad and gem related GTP binding } \\
\text { protein } 1\end{array}$ & $\begin{array}{l}\text { GTP binding, GTPase activity, } \\
\text { nucleotide binding }\end{array}$ \\
\hline zgc:153154 & prostaglandin D2 synthase-like & $\begin{array}{l}\text { small molecule binding, transporter } \\
\text { activity }\end{array}$ \\
\hline mespb & mesoderm posterior ba & $\begin{array}{l}\text { protein dimerization activity, sequence- } \\
\text { specific DNA binding transcription } \\
\text { factor activity }\end{array}$ \\
\hline zp2.3 & zona pellucida glycoprotein 2.3 & carbohydrate binding \\
\hline zgc:152898 & two pore segment channel 2 & $\begin{array}{l}\text { NAADP-sensitive calcium-release } \\
\text { channel activity, }\end{array}$ \\
\hline $\operatorname{six} 7$ & sine oculis homeobox homolog 7 & $\begin{array}{l}\text { DNA binding, sequence-specific DNA } \\
\text { binding transcription factor activity }\end{array}$ \\
\hline $\mathrm{cdx} 1 \mathrm{a}$ & $\begin{array}{l}\text { caudal type homeo box } \\
\text { transcription factor } 1 \mathrm{a}\end{array}$ & $\begin{array}{l}\text { DNA binding, sequence-specific DNA } \\
\text { binding }\end{array}$ \\
\hline
\end{tabular}

In conclusion, the maternal glucocorticoid receptor transcript dosage should be regarded as the major integral sensor of the stressor load on the mother's life during oogenesis while the cortisol deposit would act as an ancillary sensor. 


\section{Acknowledgement}

The authors would like to acknowledge Prof. L. Colombo, Dr. L.D. Valle and Dr. F. Benato for their kind cooperation and inspiration to conduct this study.

\section{References}

1. Shiraishi K, M Matsuda, T Mori and T Hirano 1999. Changes in expression of prolactin and cortisol-receptor genes during early-life stages of euryhaline tilapia (Oreochromis mossambicus) in fresh water and seawater. Zool. Sci. 16: 139-146.

2. Jesus De and T Hirano 1992. Changes in wholes body concentrations of cortisol, thyroid hormones, and sex steroids During early development of the chum salmon, Oncorhynchus keta. Gen. Comp. Endocrinol. 85: 55-61.

3. Hwang PP, SM Wu, JH Lin and LS Wu 1992. Cortisol content of eggs and larvae of teleosts. Gen. Comp. Endocrinol. 86: 189-196.

4. Campbell PM, TG Pottinger and JP Sumpter 1992. Stress reduces the quality of gametes produced by rainbow trout. Biol. Reprod. 47: 1140-1150.

5. Stratholt ML, EM Donaldson and NR Liley 1997. Stress induced elevation of plasma cortisol in adult female coho salmon (Oncorhynchus kisutch), is reflected in egg cortisol content, but does not appear to affect early development. Aquaculture 158: 141-153.

6. McCormick MI 1998. Behaviorally induced maternal stress in a fish influences progeny quality by a hormonal mechanism. Ecology 79: 1873-1883.

7. Foo JTW and TJ Lam 1993. Retardation of ovarian growth and depression of serum steroid levels in the tilapia, Oreochromis mossambicus, by cortisol implantation. Aquaculture 115: 133-143.

8. Contreras-Sanchez WM 1995. Effects of stress on the reproductive performance and physiology of rainbow trout (Oncorhynchus mykiss). MS thesis, Oregon State University. pp. 60.

9. Yeoh CG, CB Schreck, MS Fitzpatrick and GW Feist 1996b. In vivo steroid metabolism in embryonic and newly hatched steelhead trout (Oncorhynchus mykiss). Gen. Comp. Endocrinol. 102: 197-209.

10. NCBI Gene; URL: http://www.ncbi.nlm.nih.gov/gene, last accessed on 25 April 2013.

11. Thompson KL, PS Pine, BA Rosenzweig, Y Turpaz, and J Retief 2007. Characterization of the effect of sample quality on high density oligonucleotide microarray data using progressively degraded rat liver RNA. BMC Biotechnol. 7: 57.

12. Pikulkaew S, F Benato, A Celeghin, C Zucal, T Skobo, L Colombo and LD Valle 2011. The knockdown of maternal glucocorticoid receptor mRNA alters embryo development in zebrafish. Dev. Dyn. 240: 874-889.

13. Li M, SK Russell, JS Lumsden and JF Leatherland 2011. The influence of oocyte cortisol on the early ontogeny of intelectin and TLR-5, and changes in lysozyme activity in rainbow trout (Oncorhynchus mykiss) embryos. Comp. Biochem. Physiol. B 160:159-165. 